\title{
Transmission Measurements With The Beckman Quartz Spectrophotometer
}

\author{
By Kasson S. Gibson and Margaret M. Balcom
}

\begin{abstract}
The Beckman quartz photoelectric spectrophotcmeter, covering the wavelength range from about 200 to 1,200 millimicrons, is proving of great utility in spectral transmission and absorption measurements. Essential features of the instrument are described, including a constant-temperature enclosure (for samples) designed and constructed at the Bureau. Various instrumental characteristics are noted and possible errors discussed, particularly those that may be present in the measurement of polarizing samples. Wavelength calibrations are shown for two instruments. A detailed over-all check of the reliability of data obtained on the Beckman spectrophotometer by means of glass standards of spectral transmittance shows it to give results over the wavelength range from 390 to 750 millimicrons fully as reliable as those obtained with other spectrophotometers, if indicated precautions are taken.
\end{abstract}

\section{Introduction}

In a standardization and testing institution such as the National Bureau of Standards it is essential that every means be taken to insure accuracy of results. It is further desirable that many different methods and instruments be available to handle the wide variety of testing submitted.

This is true in spectrophotometry as in other fields. Spectrophotometers are subject to many annoying and hard-to-detect errors. One can have a good instrument, make a careful wavelength calibration, reduce stray light to negligible proportions, reduce the slit widths or use homogeneous radiant energy, test the photometric scale, and control the temperature of the instrument or the sample. In this and other ways he may believe that the results obtainable over the better part of the appropriate wavelength range are highly accurate. He may also do this with other instruments of different type, covering the wavelength range in which he is interested.

He may then carefully measure the spectral transmittance ${ }^{1}$ of a glass on each instrument but

\footnotetext{
1 The terms spectral transmittance $(T)$ and spectral transmittancy $\left(T_{s}\right)$ are used as defined in the Optical Society of America Colorimetry Committee Report (J. Opt. Soc. Am. 34, 183; 1944). Spectral transmittance (p. 184 and 188 ) is thus the ratio of transmitted to incident radiant flux, evaluated for a particular wavelength of incident energy. In the present paper the term is often abbreviated simply to transmittance. Spectral transmittancy (p. 189)
}

find that the results do not agree to the expected extent. He may or may not have the time or otherwise be able to eliminate the discrepancy. For this reason it is advisable to have several different types of instruments on hand, especially if spectrophotometric standards are being issued to other laboratories to check the performance of their instruments, or if effort is being made to determine with the greatest certainty the true values of transmittance at the specified wavelengths.

With respect to the wide variety of work submitted, transmission values may range from unity down to a few hundredths of 1 percent (for example, welding glasses or filters for viewing searchlights), and these values may be desired to two or three significant figures. Also, the wavelength ranges of all spectrophotometers are limited, and values obtained near the ends of these ranges are usually of lowered reliability. Furthermore, most spectrophotometers are de-

is the ratio of the transmittance of a solution to that of the solvent in equivalent thickness, again evaluated for a particular wavelength. It can be measured if the solution and solvent are placed in identical absorption cells.

Consistent with the above, the word transmission has been discontinued as a specific term, along with many other words ending in ion. As an adjective, however, the word is often useful when it is desired not to be specific. For example, in the present paper, the word transmission is used where the statement being made would apply both to the measurement of transmittance and to the measurement of transmittancy. 
signed primarily for special purposes and will handle adequately only a limited variety of testing.

For reasons such as these, the Photometry and Colorimetry Section of the National Bureau of Standards maintains several types of spectrophotometers that have been developed by the Bureau or purchased over a period of years. The development or operation of these has been described in numerous publications listed in Bureau Letter Circulars LC398, 628, and 789, and reference to some of these instruments is made later in the paper.

Two of the Beckman quartz spectrophotometers have been obtained by the Photometry and Colorimetry Section, one in 1943 and one in 1945. These were not obtained for the purpose of "testing" the instruments but to facilitate the large amount of standardization and testing work of the Section, especially during the war periods. A complete study of the instruments therefore has not been made, but sufficient information on their calibration and operation has been obtained to be of interest and value to others, as was true earlier with the General Electric recording spectrophotometer. $^{2}$

The scope of the present paper is limited mostly to transmission measurements, although much of the information obtained is equally useful in reflection work. Furthermore, only the optics of the measurements are considered, little study having been made of the electric circuits of the Beckman spectrophotometer. A few of the points noted were briefly considered in a recent publication. ${ }^{3}$

\section{Description and Operation of the Instrument}

The Beckman spectrophotometer has been discussed in some detail in a paper by Cary and Beckman. ${ }^{4}$ In that paper questions of design, stray radiant energy, phototube sensitivity, electric circuits, and slit widths are considered and illustrated. Figure 1 of the present paper is a reproduction of figure 3 of the paper by Cary and Beckman.

\footnotetext{
${ }^{2}$ Kasson S. Gibson and Harry J. Keegan, Calibration and operation of the General Electric recording spectrophotometer of the National Bureau of Standards, J. Opt. Soc. Am. 28, 372 (1938).

${ }^{3}$ Kasson S. Gibson, Spectrophotometers, ASTM Proc. 44, 725 (1944).

${ }^{4}$ H. H. Cary and Arnold O. Beckman, A Quartz photoelectric spectrophotometer, J. Opt. Soc. Am. 31, 682 (1941).
}

Radiant energy from an incandescent lamp or other illuminant, $A$, is focused on the slit, $D$, by means of the concave mirror, $B$, and the plane mirror, $C$. The beam entering the slit is collimated by mirror $E$ and passes through the quartz prism to the reflecting surface, $F$. After reflection at $F$ the beam returns along nearly the same path to the same slit, $D$, where it emerges slightly above the entrance beam and mirror $C$. After passage through the sample or sample compartment, $G$, the beam is incident on the phototube, $H$.

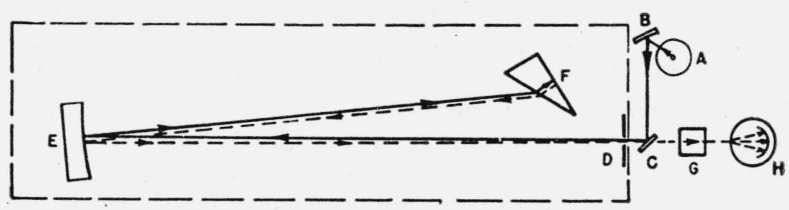

FiguRE 1.-Optics of the Beckman spectrophotometer. This is a reproduction of figure 3 of the paper by Cary and Beckman, J. Opt. Soc. Am. 31, 682 (1941).

Two phototubes are supplied installed in the instrument, either of which may be inserted in the beam at will. One is a "cesium oxide" phototube ("red sensitive") for use primarily in the range from 620 to $1,200 \mathrm{~m} \mu$. The other is a special "cesium antimony" phototube ("blue sensitive") for use primarily below $620 \mathrm{~m} \mu$. If incandescent lamp and common glass phototube are used, this range terminates at about $320 \mathrm{~m} \mu$. If the tube has an envelope of Corning 9740 glass, and if a hydrogen lamp with a window of the same type of glass is used as source (all supplied with the instrument when desired), the range extends to about $210 \mathrm{~m} \mu$.

The photometric scale is based on electrical rather than optical principles. The photoelectric current from the irradiated cell produces across the phototube load resistor a voltage drop which is balanced by a potentiometer. While this null setting is being made any imbalance is amplified electronically and is indicated by a milliammeter on the instrument. Accuracy of the photometric scale depends, among other things, on conformity of the phototube load resister to Ohm's law, and on the linearity of the irradiation-current relationship. As vacuum phototubes are used, the relation should hold closely. It is understood that linearity is still further assured by the use of a frame-type anode in the phototubes.

In general, three readings are necessary to make a transmission determination at any wavelength, 
(1) the zero reading, in which the beam is blocked from the phototube by an "off" shutter, and the galvanometer is brought to balance by a "dark current" adjustor, (2) the 100-percent reading, in which the beam falls on the phototube after passing through the transmission standard (blank beam, solvent cell, or standard glass), and the milliammeter is brought to balance by adjusting the slit width or the "sensitivity" adjustor, and (3) the transmission reading, in which the unknown or test sample is placed in the beam and the galvanometer, is brought to balance by turning the potentiometer dial carrying the "transmission" or "density" 5 scale. The 100 -percent reading may be made either by setting the dial to 100.0 or, more conveniently, by setting a selector switch to "check". This switch is then set to " 1 " for the the transmission reading, or to ". 1 " if it is desired to read transmission values between 0.0000 and 0.1000 on a " $\times 10$ " scale. Unless the transmission values are low (below 0.10), the zero reading does not usually have to be checked at every wavelength.

The instrument is designed primarily to measure the transmittancy, $\mathrm{T}_{s}\left(\mathrm{or}-\log _{10} T_{s}\right)$ of solutions relative to solvent in a l-cm thickness, and a holder is supplied for carrying the 1-cm cells furnished with the instrument, as well as shorter cells, which are also available. To measure the transmittance of glasses, it was necessary to construct a special holder that would fit into the shifting platform. The glass can thus be readily moved in and out of the beam. Samples up to about 2 inches square can be accommodated.

To handle solutions up to $10 \mathrm{~cm}$ in thickness the manufacturers supply an alternative sample chamber which may be placed between the instrument slit and the phototube compartment. They also supply absorption cells 2, 5, and $10 \mathrm{~cm}$ in length with adjustable holder. This auxiliary equipment was purchased with the first Beckman spectrophotometer, but for reasons of convenience, the carrier has been replaced by a constant-temperature enclosure designed in the Photometry and Colorimetry Section, which will accommodate the absorption cells used with the Bureau's KönigMartens and Gibson spectrophotometers.

\footnotetext{
5 The words "transmission" and "density" are engraved on the dial on which the scales are carried. By density, in this case, is meant the negative logarithm of transmittance or transmittancy.
}

\section{Constant-Temperature Enclosure}

This constant-temperature enclosure is somewhat similar to those used on the above spectrophotometers. The outside dimensions were necessarily smaller, however, as it had to fit light-tight into the sample chamber supplied with the Beckman instrument.

Figure 2 shows this enclosure along with various other accessories related to the instrument or the measurements. The temperature of the holder, $A$, is kept constant at $25^{\circ} \mathrm{C}$ or other desired temperature by means of water pumped through

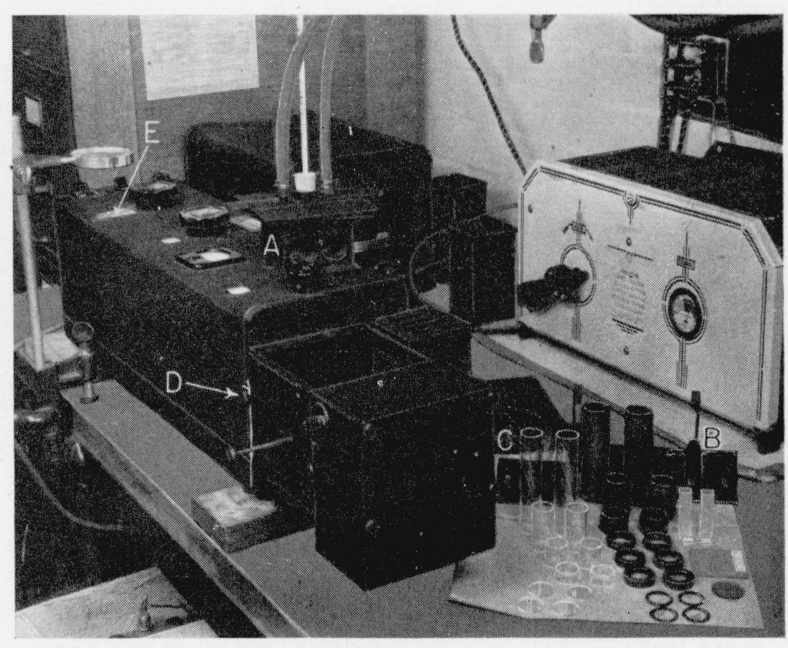

FIGURE 2.-Beckman spectrophotometer and various auxiliary equipment as installed and used in the Photometry and Colorimetry Section of the National Bureau of Standards (see text for explanation).

from a constant-temperature bath. The temperature of the water in the holder is indicated by the thermometer shown, the bulb of which is placed inside between the two cell compartments.

The absorption cell parts are shown beside the instrument. These cells are $1,2,4$, or $10 \mathrm{~cm}$ in length. They have been described in detail in previous publications. ${ }^{6}$ They consist of glass tubes on the inside, with separate glass or quartz end plates held on by the brass caps. The cells are thus easily taken apart for cleaning.

The diameter of the openings in the constanttemperature enclosure is about $30 \mathrm{~mm}$, just large enough to hold the absorption cells shown and also

\footnotetext{
${ }^{6}$ K. S. Gibson, H. J. MeNicholas, E. P. T. Tyndall, M. K. Frehafer, W. E Mathewson, The spectral transmissive properties of dyes. I. Seven permitted food dyes in the visible, ultra-violet, and near infra-red, BS Sci. Pap. 18, 121 (1922) S440. Also described in RP30 (see footnote 12).
} 
the glass standards of spectral transmittance which the National Bureau of Standards issues. Other samples, such as 2-inch squares of glass or plastic, can be mounted on the outside of the holder resting on the platform and held in place by a spring finger.

This means of temperature control is considered adequate for the purpose, which is primarily to hold the samples at some specified temperature within room-temperature range. In Washington, D. C. these room temperatures may vary from less than $20^{\circ} \mathrm{C}$ in the winter to about $35^{\circ} \mathrm{C}$ in the summer, and such variations may have important effects on transmission values. An additional reason for the temperature control is that the lamp sources on the Beckman spectrophotometer are closely adjacent to the sample compartment, and without the control, the whole enclosure (with samples) slowly heats up during extended measurements.

Although this means of temperature control is primarily for maintenance at $25^{\circ} \mathrm{C}$, it is also considered adequate for maintaining the absorption cells and $30-\mathrm{mm}$ glass standards at temperatures from about $25^{\circ}$ up to $55^{\circ} \mathrm{C}$, and has often been so used.

Certain other points of interest may be noted in figure 2. At $B$ is the holder for glass samples to which reference has already been made, used when temperature control of the sample is unnecessary. At $C$ is the four-compartinent solution-cell holder supplied with the instrument. At $D$ is the slide carrying the stray-energy filter, supplied with the instrument.

At $E$ is an auxiliary indicator line which has been added above the wavelength scale of the instrument. The use of a single indicator line, as installed in the instrument, results in parallax and consequent uncertainties of wave length setting larger than desired. A slide-rule indicator glass fits nicely into the space, and was cemented onto the instrument glass with the black line up. The two fine lines thus presented greatiy increase the precision of wave-length settings.

\section{Multiple Reflections}

Certain other points regarding the instrument and its operation may be noted. On the 1943 instrument both slits were covered with plane quartz plates to keep out dust and fumes. However, the normal divergence of the exit beam pre- vented accurate measurements with $10-\mathrm{cm}$ cells, the beam hitting the sides of the cell near the far end. To remedy this the quartz plate over the exit slit was changed to a lens of $30-\mathrm{cm}$ focal length, which rendered the beam more or less "parallel" and made it possible to use cell lengths up to $10 \mathrm{~cm}$. On the 1945 instrument, the quartz plate over the exit slit had been replaced by this type of lens when received.

On both instruments this lens has been placed in the sliding piece carrying the stray-energy filter, so that it can be moved in or out at will, and no plate or lens has been left over the exit slit. This is to avoid the slight multiple-reflection error obtained with the quartz plate or lens in use when a glass sample is measured relative to a blank beam. For a nonabsorbing glass sample of index 1.7, the error in $T$, by measurement, amounts to about 1 percent, the value obtained being too high. When solutions are measured relative to solvents in similar cells, or when one glass is measured relative to another, this effect is usually of negligible importance. Further troubles originating in the quartz plates are discussed below.

\section{Stray Spectral Energy}

Stray light can readily be perceived by looking directly into the exit slit of the instrument at a region of low luminosity - for example, below 450 or above $700 \mathrm{~m} \mu$. Likew ise, in the spectral regions to which the phototubes are relatively insensitive, stray energy from the rest of the spectrum may be present in important amounts. It should be noted that, with the usual type of cesium oxide phototube, the response obtained from 1,200 to $2,000 \mathrm{~m} \mu$ on the Beckman spectrophotometer is produced almost entirely by stray energy. Enor mous errors may be obtained if the instrument is used at scale settings nominally in this region.

One stray-energy filter is supplied with each instrument, as already indicated. This is a dense purple glass, transmitting the ultraviolet freely and absorbing the visible strongly, and, as recommended, should always be used when measurements with the incandescent source are being made below $400 \mathrm{~m} \mu$. A similar (blue) filter for the region from 400 to $500 \mathrm{~m} \mu$ is advantageous for some work. In this region, erroneous values of transmittance from 0.001 to 0.005 may be obtained with nonfluorescent yellow selenium 
glasses, whose true transmittance is known to be 0.000 .

This example shows that the importance of stray energy depends not only on the spectral region but also on the type of glass or solution being measured. This is further mentioned below in connection with the measurements made on the cobalt-blue spectrophotometric standard. Additional slides have been purchased for holding stray-energy filters of varying type, which may be inserted at will in the instruments.

To avoid multiple-reflection errors, the strayenergy filter should be mounted with surfaces inclined somewhat to the plane of the sample being measured.

\section{Measurement of Fluorescent Samples}

In spectrophotometers where the radiant energy is dispersed before incidence upon the sample, an error of measurement may occur with fluorescent samples. For example, if energy of wavelength $550 \mathrm{~m} \mu$ excites reddish fluorescence, this fluorescent energy is incident directly upon the phototube and adds to the photocurrent excited by the normally transmitted or reflected energy at $550 \mathrm{~m} \mu$. The magnitude of this error depends on the ratio of fluorescent to incident energy and on the relative sensitivity of the phototube to the incident and the fluorescent energy.

The error is apparently never very large in transmission measurements because the fluorescent energy is emitted throughout the whole spherical solid angle, and only a small fraction of it is incident upon the phototube. If of importance, the error can be reduced by placing the sample as far from the photocell as possible and by the use of selective filters transmitting the incident energy freely and absorbing the fluorescent energy strongly. The error is of much greater importance in the spectral reflection measurement of diffusing samples, because here the angle of the fluorescent energy accepted for measurement bears the same ratio to the full hemispherical angle as does the reflected spectral energy itself.

\section{Other Instrumental Characteristics}

A matter of interest and importance in the use of any spectrophotometer is the relative spectral response of the receptor and the spectral purity of the radiant energy transmitted by the exit slit under normal working conditions. The former is of importance in connection with study or elimination of errors resulting from stray energy, and the latter is of importance (along with other factors) in any consideration of the absolute accuracy of spectral-transmission measurements.

Information on both of these points is given in the paper by Cary and Beckman. In their figure 4 the relative photocurrents for nominal band widths of $3 \mathrm{~m} \mu$ are illustrated, and in their figure 9 are given the minimum spectral band widths that permit scale readings to 0.1 percent. For the Bureau instruments it was desired to know the relative spectral response of each phototube when the slits are kept at a constant mechanical width and to determine the spectral band widths transmitted by the exit slit over the whole wavelength range under normal working conditions, this latter amounting to a check of Cary and Beckman's figure 9 .

\section{Spectral-Response Curves}

The relative response curves were obtained for each phototube with the incandescent illuminant in normal operation. With the selector dial set at " 1 " the slits were adjusted to give a reading of 100.0 at the wavelength of maximum response for each cell. Readings on the photometric scale were then made at the various wavelengths through the spectrum, continual check (and slight adjustment if necessary) being made to keep all readings truly relative to 100.0 at the respective wavelengths of maximum response. When the relative response fell to about 10.0 the selector dial was changed to ".1", and the readings were made to one more decimal place. The results are shown in figure 3. Readings were taken at every 2 or $10 \mathrm{~m} \mu$, as there shown. The data for the blue-sensitive phototube were obtained with the 1943 instrument; those for the red-sensitive phototube with the 1945 instrument. Data obtained with different phototubes are of course somewhat different, but those shown are considered typical. It may be repeated that these data are for constant mechanical slit width and are not corrected for dispersion of the prism or other selectivity within the instrument or for energy distribution of source. 


\section{Polarization Effects}

It may be noted that some of the curves of figure 3 are smooth and typical of the response curves ordinarily obtained with phototubes of these kinds with incandescent illuminant and prismatic dispersion. The other curves, however, are characterized by ripples superposed on the response curves. This is shown by curves 1 and 3 of figure 3 .

The primary factor causing these ripples is the quartz plate over the entrance slit. This plate on both instruments was found to be cut with its faces parallel to the optic axis of the quartz crystal. ${ }^{7}$ When such a plate is placed between polarizing elements and oriented so that its axis is not parallel with that of either of the polarizing elements, the spectrum contains a number of dark bands regularly spaced throughout. The conspicuousness of these bands depends on the degree of polarization of the polarizing elements and on the deviation of the optic axis of the quartz plate from the polarization planes of these elements, being greatest if at $45^{\circ}$ to both of these planes.

The following explanation of the effect is given by R. W. Wood. When plane polarized light is

\footnotetext{
` Determinations made by F. P. Phelps of the Bureau's Polarimetry
} Section, from whom helpful advice in this connection was received. passed through a doubly refracting crystalline plate cut parallel to the axis, "it is resolved into two components vibrating at right angles to each other which traverse the plate with different velocities and consequently emerge with a phasedifference depending on the thickness of the plate ... Owing to dispersion the phase-difference of the emergent components depends not only on the thickness of the crystal plate but also on the wavelength of the light, consequently if white light is employed the state of polarization of the transmitted light varies as we pass along the spectrum, and if a plate of moderate thickness is used and the light is passed through a Nicol and into a spectroscope, the spectrum will be found to be furrowed by dark bands which represent the wavelengths quenched by the Nicol." 8

In the present instance the polarizing elements appear to be (1) the incandescent filament itself (which may give up to 20 -percent polarization ${ }^{9}$ ), modified possibly by elliptical polarization by the two mirrors, on one side of the quartz plate, and (2) the refracting surface of the dispersing prism, on the other side.

\footnotetext{
${ }^{8}$ Physical optics, 3d ed., p. 349 (The Macmillan Co., New York, N. Y. 1934).

${ }^{9}$ A. G. Worthing, Deviation from Lambert's law and polarization of light emitted by incandescent tungsten, tantalum, and molybdenum and changes in the optical constants of tungsten with temperature, J. Opt. Soc. Am. 13. 635 (1926).
}

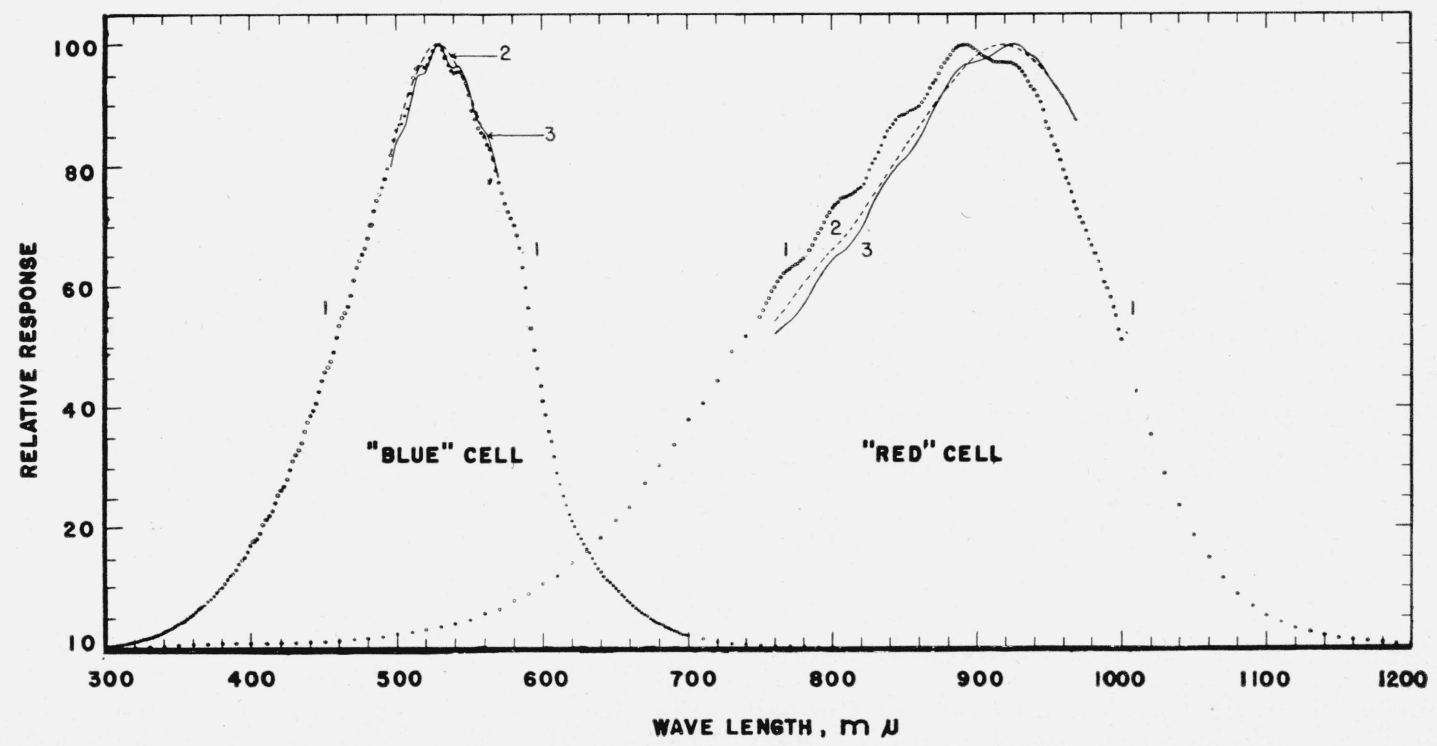

FIGURE 3.-Spectral-response curves of phototubes in a Beckman spectrophotometer.

These are as obtained, relative to 100.0 at the respective maxima, for constant mechanical slit widths and with no corrections for energy distribution of source, dispersion of spectrometer, or other factors affecting the selectivity. For explanation, see text. 
The ripples in the sensitivity curves are not apparent if (1) the quartz plate is oriented with its axis at $0^{\circ}$ or $90^{\circ}$ to the polarizing plane of the dispersing prism, (2) the plate is removed from the instrument, (3) a quartz plate cut, perpendicular to the axis is used over the entrance slits, or (4) a glass plate is used over the entrance slit. The quartz lens or plate in the upper beam (regardless of how cut) has no appreciable effect on the sensitivity curve at any orientation; in this case, the quartz is not between polarizing elements.

The band effect can readily be seen if the quartz plate (faces cut parallel to optic axis) is taken out of the instrument and examined between polarizing elements with a hand spectroscope. One of the polarizing elements may be the sky or a bare tungsten filament. The filament of the incandescent source of the Beckman spectrophotometer is thus demonstrated to be giving off partially polarized light. The dispersing prism of the hand spectroscope is the second polarizing element. Of course, the effect is far more pronounced if the polarizing elements are two Polaroids or nicols.

With reference to figure 3 , again, curves 1 were obtained soon after the receipt of the respective instruments. In both cases the entrance quartz plate had not been disturbed before the data were taken, and it would appear from the magnitudes of the ripples that each plate had been mounted with its axis approximately at $45^{\circ}$ to the polarizing planes of the dispersing prism and lamp filament. The other curves of figure 3 were obtained later, curves 2 with the entrance quartz plate removed, curves 3 with it reinserted at the $45^{\circ}$ position. It may be noted, incidentally, that curves 1 and 3 ("blue" cell), which check closely, were obtained with different phototubes; curves 1 and 3 ("red" cell), which show change of sensitivity, were obtained with the same phototube 14 months apart.

It was at first thought that the ripples shown in figure 3 were of no particular importance. However, when measurement of the spectral transmittance of polarizing materials was attempted, it was found that the ripples were caused by some polarization phenomena, and could no longer be ignored. The effect was accordingly investigated, as partially described above. It is unnecessary to consider all the data obtained, but it is worthwhile to point out some of the results obtained if due precautions are not taken.

For example, in figure 4, top, is shown (1) the "transmittance" curve obtained on a polarizing goggle inserted in the carrier of the Beckman spectrophotometer at random orientation ${ }^{10}$ and with the entrance plate of the instrument at unknown orientation. From 570 to $600 \mathrm{~m} \mu$ are shown also (2) the maximum and minimum values of "transmittance" obtained by turning the goggle in its own plane $\left(90^{\circ}\right.$ apart), and (3) the corresponding values obtained on the Gibson photoelectric spectrophotometer (with Hilger constant-deviation glass prism). Similar ripples have been noted in the transmission curves of polarizing materials obtained by others with Beckman quartz spectrophotometers.

The effect was studied at some length by measuring the "transmittance" of a Polaroid sample, these measurements more or less paralleling in time those from which the sensitivity curves were obtained, as described above. Some of the data are presented in the lower half of figure 4.

In figure $4 A$, the quartz plate over the entrance slit is placed with its optic axis at $45^{\circ}$ to the slit, and no plate or lens is over the exit slit. The angles shown beside the five curves in $A$ refer to various orientations of the polaroid. In the $0^{\circ}$ position the electric vector of the radiant energy transmitted by the Polaroid is in the vertical plane parallel to the slits and the refracting faces of the dispersing prism. At $+45^{\circ}$ and $-45^{\circ}$ the Polaroid is turned at $45^{\circ}$ from the $0^{\circ}$ position. At $+90^{\circ}$ and $-90^{\circ}$ the Polaroid is turned at right angles from the $0^{\circ}$ position.

In $B$ and $C$, figure 4 , the quartz entrance plate has been turned so that the optic axis is respectively horizontal and vertical, the angles on the graphs indicating the same respective orientations of the Polaroid as before. No plate or lens is over the exit slit.

In $D$, figure 4 , the quartz entrance plate has been removed, and there is no plate or lens over the exit slit. For comparison are shown (the large crossed circles) the corresponding values obtained on the Gibson glass-prism spectrophotometer.

In $E$, figure 4 , the quartz plate is still removed from the entrance slit but has been inserted over the exit slit at the $45^{\circ}$ position. Data were also obtained with the quartz plate over the exit slit oriented at $0^{\circ}$ and $90^{\circ}$. At $0^{\circ}$ the curves obtained

${ }_{10}$ The shape and size of the sample did not permit of orientation at $45^{\circ}$. 
were similar to the Beckman (small circle) curves of figure $4, D$, with about the same total spread. At $90^{\circ}$ the curves were similar to those of figure $4, C$, but with a total spread of only 0.02 .

The following comments may be made regarding the data of figures 3 and 4 .

1. The conditions causing the ripples of figure 3 are the same as those causing the worst distortions, figure $4, A$, during measurement of the transmittance of polarizing samples. It is probable that the entrance quartz plate was close to the $45^{\circ}$ position when the data shown in the top half of figure 4 were obtained.

2. Complete elimination of the trouble is effected by removing both quartz plates (or plate and lens) over the entrance and exit slits, figures 3 and $4, D$.

3. Residual effects not apparent in the sensitivity curves may be found (by means of the Polaroid) under other than $45^{\circ}$ orientation of the entrance quartz plate (cut parallel to the optic axis). Such residual effects are shown in figures $4, B$ and $C$, and in other data not given. In this connection it should be pointed out that the angles indicated in figure 4 were rather crudely determined, particularly the angles at which the quartz plate was oriented. They are subject to an uncertainty of at least $\pm 5^{\circ}$. Whether or not, for example, the "ripples" of figure $4, B$ and $C$, would be eliminated by more exact $0^{\circ}$ and $90^{\circ}$ positioning was not investigated.

4. With both quartz plates removed the errors possible in the measurement of a polarizing sample at random orientation appear much less than on a glass-prism instrument. Note in figure $4, D$ the total spread of about 0.02 on the Beckman as contrasted with the spread of about 0.11 on the Gibson instrument. This is undoubtedly largely due to difference in the angle at which the radiant energy enters and leaves the respective quartz and glass prisms at $530 \mathrm{~m} \mu$ on the two instruments.

Many of these results were shown to Mr. Cary (see footnote 4) in November 1946, who indicated that the entrance plates and the exit lens or plate were cut from wastage material remaining from construction of the quartz dispersing prisms and that the optic axis in these plates may be parallel or perpendicular to the faces or at angles in between. Accordingly, other users of the Beckman may or may not have been bothered by the effects shown in figures 3 and 4 . As, in the past, these plates have thus been cut at various
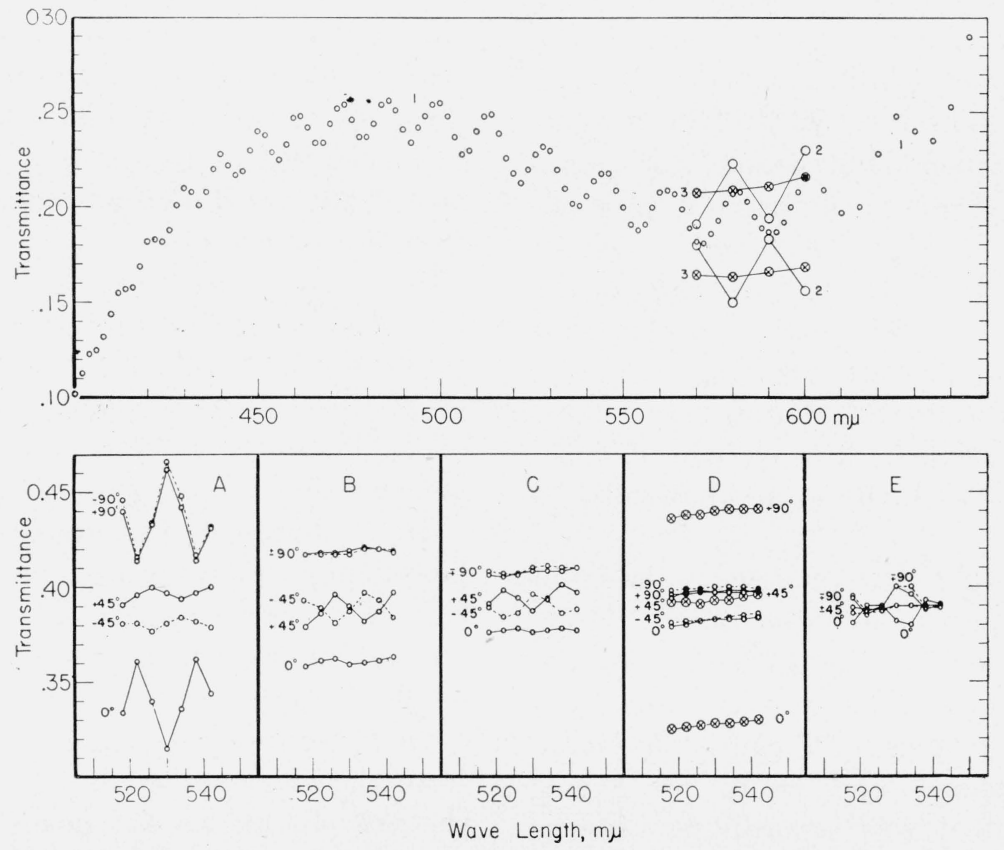

FIGURE 4.-Effect on the measured "transmittance" of polarizing materials caused by a quartz" plate cut with optic axis parallel to surfaces when placed before entrance or exit slit at various orientations (for explanation, see text). 
angles to the optic axis and apparently inserted at random, it seems well to state the following conclusions:

1. No trouble has been experienced and no errors detected relating to this effect, except in the measurement of polarizing materials.

2. To avoid important errors in the measurement of polarizing materials, the entrance quartz plate has been removed and the exit quartz lens is used only when it is necessary or desirable to collimate the beam (as when measuring thick samples or in reflection work).

3 . One can readily study the effect on his instrument by carrying out measurements similar to those shown in the lower half of figure 4. By measuring the transmittance of a polarizing sample at various orientations over a short range of wavelengths, the effect can be quickly found if present.

\section{Spectral-Purity Data}

The spectral purity - that is, the extent of spectrum transmitted by the slits as normally used-was obtained as follows: For any given set of conditions (source, filter, phototube), with the "sensitivity" dial set three turns counterclockwise from extreme (as recommended in the instructions), and with the selector switch set at "check" (equivalent to 100.0 on the " 1 " scale), the slit widths in millimeters necessary to obtain a balance of the galvanometer were determined. The mechanical slit widths so determined were then multiplied by the values of millimicrons per millimeter supplied by the manufacturer.

The results are shown in figure 5. These values were obtained on the 1943 instrument. Values obtained on the second instrument are somewhat greater but otherwise similar. In either case the values are comparable with those illustrated by Cary and Beckman in their figure 9.

These slit widths are in general smaller, in some cases much smaller, than are usually practicable with commercial instruments and comprise one of the major advantages of the Beckman spectrophotometer for certain types of work. It is pointed out by the makers of the instrument that still smaller slit widths may be used if the 100 -percent reading is made with the selector switch set at " 1 " instead of at "check". The sensitivity is, of course, decreased under this condition.

The narrowness of the slits makes unimportant for much work the fact that the operator of the instrument is limited in his freedom to set the slits at any desired width, and in normal operation they will vary as shown in figure 5. For most materials, for example, essentially the same result will be obtained whether the slits have widths equivalent to 1 or $2 \mathrm{~m} \mu$. However, with many of the rareearth glasses or solutions, this is not true. Although, in general, the narrower the slits the more nearly will the measured transmission value approach the true transmission value, even on the Beckman instrument the slits are not narrow enough to make such further change negligible

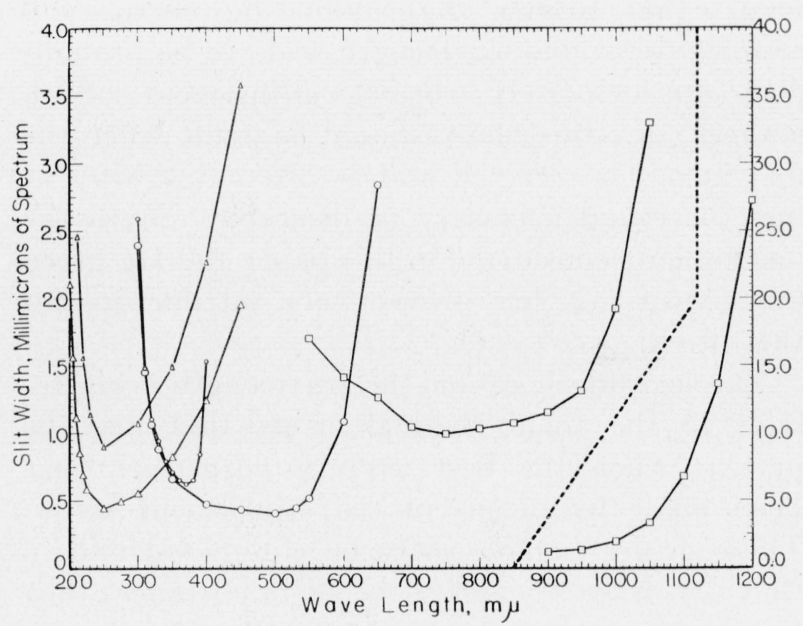

FiguRE 5.-Approximate amounts of spectrum transmitted by the slits of a Beckman spectrophotometer under normal conditions of operation.

$\triangle$, Hydrogen, new; $\triangle$, hydrogen, $6 \mathrm{hr}$ operation; $\bigcirc$, incandescent, "blue" cell, with filter; $\bigcirc$, incandescent, "blue" cell; $\square$, incandescent, "red" cell.

with these materials. This is of considerable importance in chemical analytical work. In such work, in comparing results on different instruments or in analytical work with any instrument, it may be more important that the results be obtained at known slit widths than that these widths be as narrow as possible.

\section{Wavelength Calibration}

The wavelength scale of the Beckman spectrophotometer provides direct reading in millimicrons. Because of the variable dispersion incident to all prism spectrophotometers, this results in greatly varying lengths of the intervals on the scale representing any given number of millimicrons as the spectrum is traversed from the ultraviolet through the visible and into the infrared. On the Beck- 
man spectrophotometer the smallest scale division is equal to $0.5 \mathrm{~m} \mu$ from 200 to $300 \mathrm{~m} \mu, 1 \mathrm{~m} \mu$ from 300 to $400 \mathrm{~m} \mu, 2 \mathrm{~m} \mu$ from 400 to $600 \mathrm{~m} \mu$, and 5 $\mathrm{m} \mu$ from 600 to $2,000 \mathrm{~m} \mu$. (However, as pointed out elsewhere, the sensitivity of the phototube does not permit reliable measurement above 1,200 $\mathrm{m} \mu$, approximately.)

On a direct-reading spectrophotometer two types of wavelength checks should be considered if the greatest accuracy with the instrument is to be obtained. On the Beckman instrument one of these checks relates to adjustment of the internal screw (reached from the left end of the instrument) that rotates the collimating mirror and enables the whole wavelength scale to be brought into approximately correct adjustment. Such a check, or adjustment, should be made when the instrument is received and as often thereafter as may be found necessary or desirable. With the instruments considered in this paper the $\mathrm{Hg}$ green line $(546.1 \mathrm{~m} \mu)$ has proved very satisfactory for this check.

The second check on the wavelength scale relates to the small residual errors that may be present when the best average adjustment has been made by means of the adjustment screw. These errors cannot easily be eliminated and, if they are large enough to be of importance, they should be allowed for in setting the wavelength scale.

Four light sources have been used in the wavelength calibration of the instruments: (1) the quartz-mercury lamp supplied with the Beckman spectrophotometer if desired, (2) the hydrogen arc, also supplied with the instrument if desired, (3) a helium lamp (vacuum tube), and (4) a similar neon lamp.

A calibration technic was employed that gave scale corrections always on the basis of correct adjustment (zero error) at $546.1 \mathrm{~m} \mu$. In effect, settings with this $\mathrm{Hg}$ reference line were made before and after the settings with each of the numerous other lines of the various sources, 10 settings being made on each. Any deviations of the settings on the reference line from the correct value of 546.1 were allowed for in determining the deviations for the other line. In making this allowance the relative variable dispersion of the prism (as represented by the millimicrons-permillimeter slit-width curve supplied by the makers) was taken into account.
The scale corrections thus obtained are shown in figure 6 for the two instruments. It will be noted that the $\mathrm{Hg}$ green line at 546.1 (the "Ref." point) has zero correction in both plots.

In studying these correction curves the millimicron equivalent of 0.1 scale division should be noted; this is shown between the two correction curves. This equivalent is also important as affecting the precision of the settings; note parti-

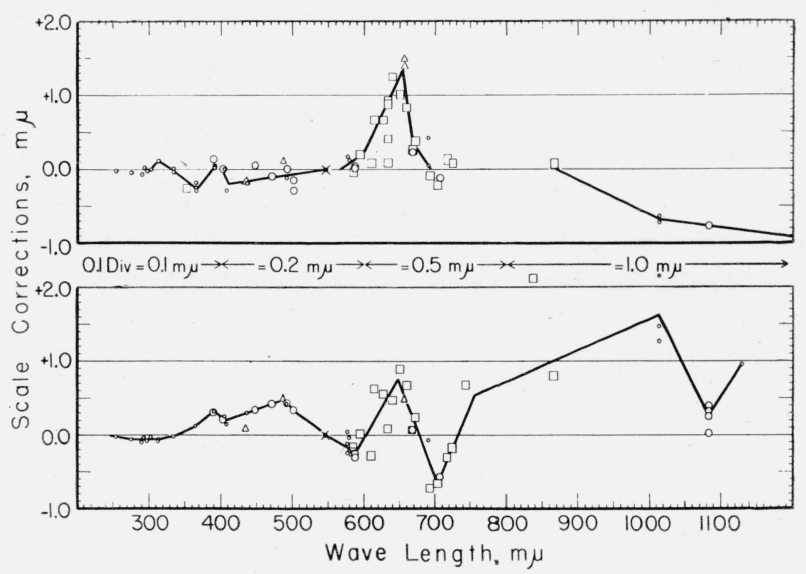

FiguRE 6.-Residual wavelength corrections found on two Beckman spectrophotometers after adjustment of each instrument to have zero correction at 546.1 millimicrons.

$$
\text { , Hg; } \bigcirc \text {, He; } \square, \mathrm{Ne} ; \triangle \text {, H; X, Ref. }
$$

cularly the difference in precision above and below $600 \mathrm{~m} \mu$.

These data are published with the thought that they may serve to inform others of the magnitudes of the wavelength errors to be expected on the Beckman spectrophotometers. They are so small that they may probably be neglected in most work for which the instruments are used. They are likewise so small that they can be determined only by the most careful work and after installing the auxiliary indicator line over the wavelength scale (see p. 604) to eliminate parallax. On the other hand these errors are large enough to be important in much of the Bureau's work, particularly when standardization is being done.

Whether or not the detailed check illustrated in figure 6 is made, it may be emphasized that check should often be made on some reference line to insure that gross wavelength errors have not developed. 


\section{Check of the Photometric Scale}

A check of the photometric scale of a spectrophotometer independent of all other sources of error is difficult or impossible to make on most spectrophotometers. Useful for tbis purpose would be a series of samples whose respective transmittances do not vary with wavelength, which will not displace the beam, and for which the transmittances can be independently determined with high accuracy. No such glasses or other materials are available, however.

On a visual spectrophotometer such a check is possible by means of rapidly rotating sectors. If such sectors are properly made, the fraction of the total opening relative to $360^{\circ}$, that is, the effective transmittance of the sector, can be measured on a circular dividing engine with uncertainties only in the fifth decimal place. Of course, the use of such rotating sectors is based on the validity of Talbot's law. Throughout the spectrum, no certain deviations from this relation are known, however. As a matter of fact, average values obtained over a period of years in measuring the "transmittances" of accurately calibrated rotating sectors on the NBS König-Martens spectrophotometer prove both the validity of Talbot's law at various wavelengths and the reliability of the instrument over most of the photometric scale, or else there is a remarkable balancing of errors.

On photoelectric spectrophotometers, however, at least the ones considered in this paper, the use of rotating sectors to check the reliability of the photometric scale is either impossible or attended with too much uncertainty for one reason or another.

Accordingly, shortly after the advent of commercial photoelectric spectrophotometers, the National Bureau of Standards instituted the service of issuing glass standards of spectral transmittance. ${ }^{11}$ To date, about 80 of these filters have been issued with accompanying certificates.

Three of the four types of filters used for this purpose are of moderate selectivity, covering a large part of the transmittance range from 0.00 to 0.90 at various wavelengths. A single filter thus covers a considerable part of the transmittance scale. In one sense these are inferior to strictly neutral filters in that a deviation from the true value may be due to other causes than inaccuracy of the photometric scale. On the other

${ }^{11}$ K. S. Gibson, Geraldine K. Walker, and Mabel E. Brown, Filters for testing the reliability of spectrophotometers, J. Opt. Soc. Am. 24, 58 (1934) hand, they are superior to the neutral filters in detecting stray-light, slit-width, and gross wavelength errors.

One of the four types of filters used for these spectrophotometric standards is a fairly dense cobalt blue glass, designated as Corning G55A. (This glass is also known as Corning Signal Purple, present catalog designation 5551.) The $30-\mathrm{mm}$ disks cut from the original roundel were recently exhausted, and it became necessary to prepare new samples from a new roundel. This of course required an extensive series of measurements in order that values of transmittance might be determined with satisfactory certainty. (After basic values are thus determined for selected disks from a given roundel, values of spectral transmittance for other disks cut from this roundel can be determined with much less labor, as noted below).

About the time that these measurements were necessitated, the second Beckman spectrophotometer was received. It was decided that an excellent opportunity was thus presented for (1) making a fairly thorough check of the reliability of transmittance measurements made on the Beckman spectrophotometer as it was received from the manufacturer, and (2) if no consistent errors appeared to be present in the Beckman data thus obtained, increasing the over-all reliability of the basic spectral-transmittance values for the new glass disks. Only three things were done to the second Beckman instrument for these measurement - the quartz lens over the exit slit was removed to eliminate multiple-reflection errors, a careful check of the wavelength calibration was made after installation of the second indicator line, and the temperature-control sample holder constructed at the Bureau was used, all as previously noted.

Corresponding work with the first Beckman spectrophotometer received in 1943 had not been done for two reasons: (1) the urgency of other work made it impossible to devote the necessary time for such measurements as long as the original supply of spectrophotometric standards held out, and (2) trouble was experienced in readings obtained with the cesium-oxide phototube, particularly at low transmissions, in that there was excessive and erratic "creep" of the galvanometer needle, and a definite and trustworthy reading at these low transmittances could seldom be obtained. Changing phototubes did not remedy the trouble. 
Similar trouble was not experienced with the cesium-antimony phototube or with either phototube circuit on the new instrument.

Among the many disks cut from the second G55A roundel, designated as $\mathrm{G} 55 \mathrm{~A} \beta$, were two which were identical in spectral transmittance, and a third which was also identical over part of the range. The identity of these spectral transmittances was established within about 0.1 percent of the transmittance by comparative measurements on the Beckman spectrophotometer.

Measurements were accordingly made on each of these glasses by each of the authors, on the
Beckman, the König-Martens visual ${ }^{12}$ and the Gibson photoelectric ${ }^{13}$ spectrophotometers, and at the various wavelengths in the visible spectrum indicated in table 1 . The temperature of the sample was kept at $25^{\circ} \mathrm{C}$ for all the measurements, so that this source of variation or uncertainty was eliminated. ${ }^{14}$

${ }^{12} \mathrm{H}$. J. MeNicholas, Equipment for routine spectral transmission and reflection measurements, BS J. Research 1, 793 (1928) RP30.

${ }^{13}$ K. S. Gibson, Direct-reading photoelectric measurement of spectra transmission, J. Opt. Soc. Am. \& Rev. Sei. Instr. 7, 693 (1923).

14 The General Electric recording spectrophotometers were not used in this standardization of the G55A $\boldsymbol{\beta}$ glass because (1) no temperature control has been devised for use on these spectrophotometers, and (2) the slit widths are undesirably large for this type of work.

TABLE 1.-Values of transmittance as measured with the Beckman, König-Martens, and Gibson spectrophotometers

Sample: Corning G55A $\beta$ of 3-mm thickness; temperature: $25^{\circ} \mathrm{C}$.

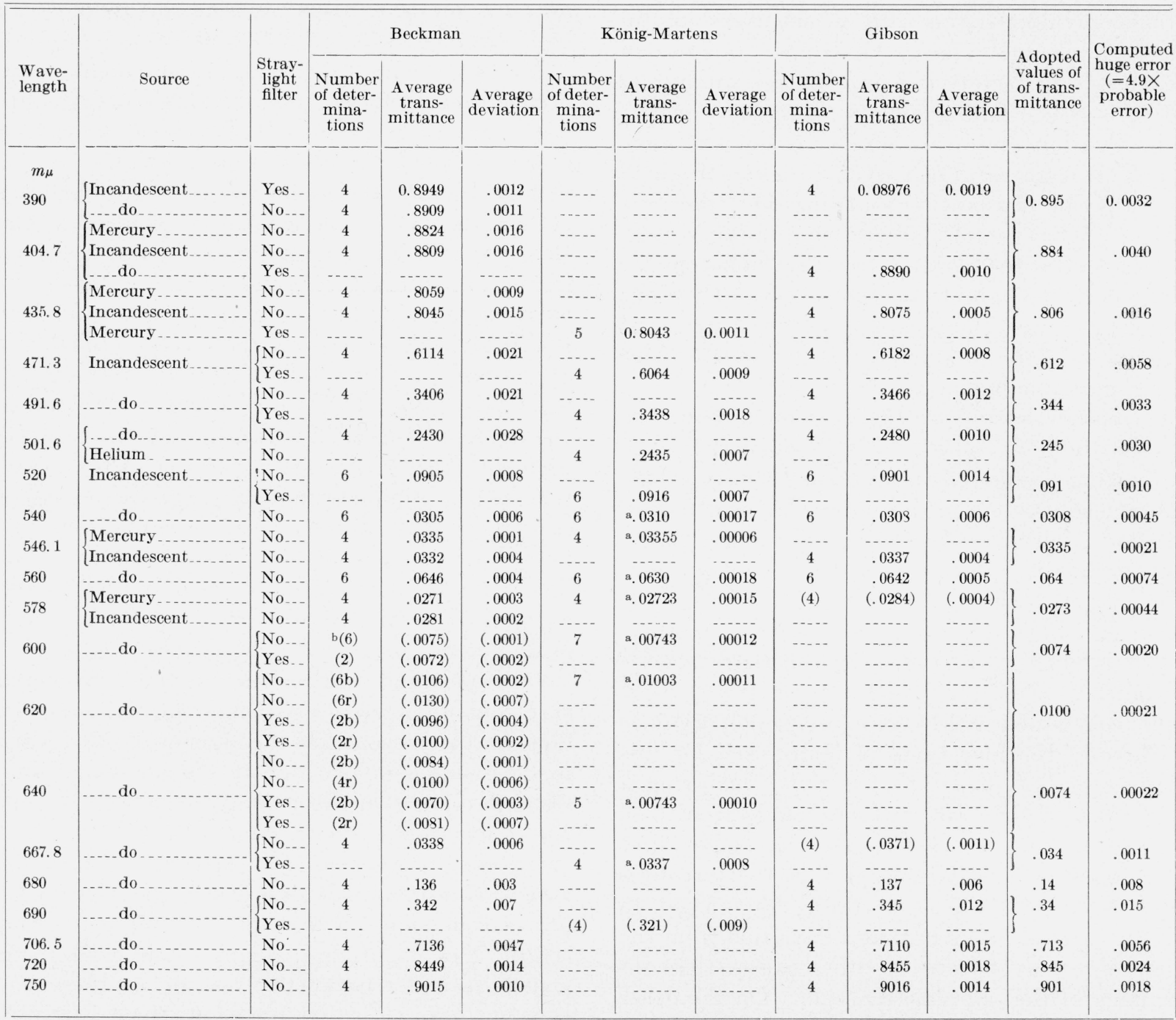

a Rotating sector used in blank beam.

b Values in parentheses were not used in deriving the adopted values of transmittance nor in computing the huge errors. 
In table 1 are given for each of the three instruments, at each of the respective wavelengths, the number of determinations of transmittance made, the average value of the transmittance so measured, and the average deviation of each determination from the average value. On the Beckman and Gibson spectrophotometers, each determination consists of two or more readings with separate wavelength settings. On the KönigMartens spectrophotometer a determination is derived from 20 photometric settings, 10 with the sample in each beam. In columns 2 and 3 and in the footnotes are indicated certain experimental conditions pertinent to the determinations. In the last two columns are given the basic values of transmittance adopted from the measurements for the NBS standard glass, together with the "huge error" (4.9 times the probable error) ${ }^{15}$ as computed from the deviations of the several separate determinations from this adopted value. Eight or more determinations always entered into the computation of this huge error.

In a consideration of the data of table 1, the following points should be noted:

1. The König-Martens and Gibson spectrophotometers were used in the usual routine manner.

2. As part of the purpose of these measurements was a test of the Beckman instrument, measurements were made at all wavelengths. On the other two instruments measurements were not made at those wavelengths which long experience has indicated to be relatively unreliable. This obviously applies to the violet and red ends of the spectrum with the König-Martens visual spectrophotometer. For this type of glass with the Gibson spectrophotometer it applies to the region from 600 to $660 \mathrm{~m} \mu$, where the transmittance is extremely low and stray energy renders the values relatively unreliable; this stray-energy error is believed to affect the values for this glass measured on this instrument at 578 and $667.8 \mathrm{~m} \mu$ also.

3. The same sort of error is apparent in the data obtained with the Beckman spectrophotometer in the region of very low transmittance with this glass, though apparently to a less extent. In both instruments this is the region in which the

15 Mansfield Merriman, A textbook on the method of least squares, 8th ed., rev., p. 205 (John Wiley \& Sons, Inc., New York, N. Y., 1915). Peter's approximation formula was used to compute probable errors. "blue sensitive" and the "red sensitive" phototubes give about the same response-near 590 $\mathrm{m} \mu$ on the Gibson and near $625 \mathrm{~m} \mu$ on the Beckman. Normally, in either case the phototube giving the greatest response is used in the measurements. For most types of samples either instrument, with the appropriate phototube, will give as accurate results through this region as at other regions. With this glass, however, for which the transmittance being measured is very low and for which the transmittance is high at those spectral regions in which the phototube is much more sensitive, the freely transmitted stray energy from these regions may produce an important error. On the Beckman spectrophotometer this was checked by the use of stray-energy filters-Corning 2424 with the "blue sensitive" phototube from 600 to $640 \mathrm{~m} \mu$ and a Corning medium Aklo with the "red sensitive" phototube from 620 to 640 $\mathrm{m} \mu$. It can be seen that the measured transmittance is lowered and on the average comes close to the values obtained with the König-Martens spectrophotometer.

4. The three instruments for which data are given in table 1 differ in the number of decimal places which can be obtained in the transmittance, $T$.

(a) Values of $T$ on the Gibson spectrophotometer can be read to only 0.001 , or at most $0.000_{5}$. However, this reading is definite as a vernier is provided on the reading scale of the rotating sector. For low transmittances the number of significant figures is therefore limited, and the fourth decimals given in the table result simply from the averaging.

(b) On the Beckman instrument, values of $T$ can be read to 0.001 by estimating tenths of a division, or on the same dial to 0.0001 for values of transmittance less than 0.11 if the selector dial is set to ".1". This greatly facilitates making the reading, but otherwise there appears to be little gain in the use of the magnified scale. Slight and erratic "creeping" of the galvanometer often makes it uncertain when the best reading is obtained. The authors have been able to obtain the precision indicated by the average deviations shown in table 1 for these low values only by consistent checking of the "off" reading, bringing the transmittance reading to such a value that the galvanometer position is identical for the two juxtaposed readings. 
(c) On the König-Martens spectrophotometer, the photometric settings are read to tenths of a degree (by vernier if desired) and transmittances computed by the cot $\times$ tan relation. The normal number of significant figures is three for transmittances greater than 0.10 , although four are often kept temporarily. When the " $10 \%$ " sector ( $T=$ 0.09945) is used in the blank beam, the same number of significant figures is obtained but the uncertainty as represented by the average deviation has been moved to the fourth decimal place. With the " $1 \%$ " sector $(T=0.00883)$ there is another displacement of the significant figures, but the lowered brightness may result in slight uncertainty still in the fourth decimal.

5. The precision or reproducibility of readings of the Beckman spectrophotometer, as judged by the average deviations, is comparable to that of the other instruments, except that the König-Martens is better than either the Beckman or the Gibson instruments at the low values of $T$, as already discussed. It should be noted that each of the four to six determinations at each wavelength on the various spectrophotometers was made on a different day, this work extending off and on over a period of weeks. The reproducibility of either photoelectric instrument at any one time and wavelength setting is in general superior to that indicated in table 1 . The Beckman is the most rapid of the three instruments, being somewhat superior in this respect to the Gibson and greatly superior of course to the König-Martens (visual).

6. No error in the photometric scale of the Beckman spectrophotometer can be demonstrated by the data of table 1 . The following details may be noted:

(1) The consistent differences between results by the Beckman and the Gibson spectrophotometers at the shortest wavelengths are not representative of results always obtained on these instruments at these wavelengths. For example, see below in connection with the results of table 2 .

(2) Except at 390 and $404.7 \mathrm{~m} \mu$, the average value at each wavelength obtained on the Beckman either lies between the values obtained on the other instruments or agrees closely with one of them. This holds even at the low values at 620 and $640 \mathrm{~m} \mu$ when the stray-energy filters are used.

(3) The use of the $\mathrm{Hg}$ source at the four wavelengths noted has proved very satisfactory. After a short warming-up period the lamp becomes steady. The slit widths are larger than with the incandescent source, but this is of no consequence as the light is homogeneous. It may be noted that in every case the values obtained with the $\mathrm{Hg}$ source are closer to the finally adopted values than those obtained at the same wavelengths with the incandescent source. The values ob. tained with the $\mathrm{Hg}$ source are not subject to wavelength error or to as great a stray-energy error.

TABLE 2.-Comparison of average transmittance obtained with the Beckman spectrophotometer with those adopted from extensive measurements on the Beckman, KönigMartens, and Gibson spectrophotometers

[Samples: Jena BG 14 (Green) and Corning HT (Yellow C), each of 2-mm thickness. Temperature: $25^{\circ} \mathrm{C}$. Measurements by Lois A. Peterson]

\begin{tabular}{|c|c|c|c|c|}
\hline \multirow{2}{*}{$\begin{array}{l}\text { Wave } \\
\text { length }\end{array}$} & \multicolumn{2}{|c|}{ Adopted transmittance } & \multicolumn{2}{|c|}{$\begin{array}{l}\text { Deviation of average } \\
\text { Beckman value from } \\
\text { adopted transmittance }\end{array}$} \\
\hline & $\begin{array}{c}\text { Jena } \\
\text { BG14 }\end{array}$ & $\begin{array}{c}\text { Corning } \\
\text { HT Yellow } \\
\text { C }\end{array}$ & $\begin{array}{l}\text { Jena } \\
\text { BG14 }\end{array}$ & $\begin{array}{c}\text { Corning } \\
\text { HT Yellow } \\
\text { C }\end{array}$ \\
\hline$m \mu$ & & & & \\
\hline 390 & 0.862 & 0.025 & 0.000 & 0.001 \\
\hline 404. 7 & .877 & .020 & .000 & -.001 \\
\hline 420 & . & .019 & . & .000 \\
\hline 435.8 & .893 & .0240 & .000 & .0003 \\
\hline 471.3 & .894 & .081 & .001 & .000 \\
\hline 501.6 & .859 & .208 & .001 & -.001 \\
\hline 520 & - n. & .316 & - & .001 \\
\hline 530 & .760 & .379 & -.001 & -.001 \\
\hline 546.1 & .671 & .479 & .001 & -.001 \\
\hline 560 & .585 & .557 & -.002 & .002 \\
\hline 578 & .473 & .636 & -.001 & .000 \\
\hline 587.6 & - & .668 & - & .000 \\
\hline 600 & .350 & .699 & -.001 & .000 \\
\hline 620 & .256 & .731 & .001 & .000 \\
\hline 640 & .187 & .747 & .001 & .002 \\
\hline 660 & - & .754 & - & .000 \\
\hline 667.8 & .122 & - n & .000 & - \\
\hline 690 & - & .755 & - & -.001 \\
\hline 710 & .074 & - & .001 & - \\
\hline 720 & - & .748 & - & -.001 \\
\hline 750 & .057 & .730 & .000 & -.001 \\
\hline 800 & .051 & .693 & .000 & .000 \\
\hline 850 & .057 & - n & .000 & - \\
\hline 900 & .072 & .631 & .001 & +.001 \\
\hline 950 & .094 & - & .000 & - \\
\hline 1000 & .124 & .600 & .001 & .000 \\
\hline
\end{tabular}

(4) At all but the lowest transmittances, the values obtained on the Beckman instrument differ from the finally adopted values by amounts that (1) are less than the uncertainties of the adopted values, and (2) do not exceed 1 percent of the value.

7. The adopted values and huge errors shown in the table are similar to those certified on the G55A standards of spectral transmittance issued by the National Bureau of Standards, except 
that in the certificates the huge errors are designated by the term "uncertainty" and are reported to the same number of decimal places as the transmittance itself.

In further confirmation of the reliability of the photometric scale of the Beckman spectrophotometer are results obtained more recently by Lois A. Peterson of the Photometry and Colorimetry Section, with two others of the NBS standards of spectral transmittance, a Jena green glass and a Corning yellow glass. The same spectrophotometers were used as for the work on the blue glass standard just described, and the number of determinations, the uncertainties, and the various other factors entering into the measurements were all comparable with those listed above. No strayenergy filter was used on the Beckman except at $390 \mathrm{~m} \mu$.

The results are shown in table 2 , in which are given for each standard (1) the adopted (usually the average) values obtained as a result of the extensive measurements on the three spectrophotometers (except that the visual determinations do not extend beyond 435.8 and $667.8 \mu$ ), and (2) the deviations of the average Beckman values from these adopted values. Examination of these data shows that there is no consistent deviation of the spectral transmittances obtained on the Beckman spectrophotometer from those obtained on the other instruments.

In addition to the general over-all reliability of the Beckman spectrophotometer demonstrated above, special note may be made of the precision and reliability with which two nearly identical samples may be spectrophotometrically compared. In this case the " $100 \%$ " reading is taken with a standard sample in the beam (instead of with the beam blank or with a solvent cell in the beam) and the only condition that has been changed to obtain balance is that the slits have been widened. Of course, the lower the transmittance the wider must the slits be for balance, but as this width will vary inversely as the square root of the transmittance they will be only about 10 times the normal width when the transmittance is as low as 0.01 .

For comparing two nearly identical samples such widths are not at all excessive. As a result, if accurately calibrated standards are available, such as the three glasses to which reference has been made above, the spectral transmittance of similar glasses may be determined in a very short time with an accuracy comparable to that of the standard, even for the very low transmittances. In other words, the comparison can be made with uncertainties of but 0.1 or 0.2 percent of the transmittance, regardless of whether this is near 0.9 or 0.01 .

An additional important feature of these narrow slits is that low transmittances can be determined by a "step-down" procedure, in which, for example, a glass transmitting 10 percent may be measured and used as standard for a glass transmitting 1 percent, that in turn for a glass transmitting 0.1 percent, and so on. On the Beckman instrument the slits do not become excessively large in this procedure.

\section{Conclusions}

On the basis of measurements made mostly on a Beckman spectrophotometer received in the Photometry and Colorimetry Section of the National Bureau of Standards in 1945, it appears that the instrument is capable of precise and accurate measurement of spectral transmittance or transmittancy. This conclusion is based on measurements of the spectral transmittance of certain glass standards on the Beckman, the KönigMartens, and the Gibson spectrophotometers. The measurements were limited to the spectral range from 390 to $750 \mathrm{~m} \mu$, but there seems no reason to doubt the reliability of transmission data obtained on the Beckman instrument at wavelengths beyond this range - to approximately $1,000 \mathrm{~m} \mu$ in the infrared, and to either 320 or $210 \mathrm{~m} \mu$ in the ultraviolet, depending on the source and phototube used in the instrument.

On all three instruments the glass samples were kept at $25^{\circ} \mathrm{C}$, being mounted in temperaturecontrol boxes constructed at the National Bureau of Standards. The general statement of reliability given above is also dependent on careful check of the wavelength scale and on adjustment or correction of any errors greater than about $0.1 \mathrm{~m} \mu$. Furthermore, it applies only to values of transmittance from 1.00 to about 0.02 . Below 0.02 there is no demonstrated error in the photometric scale, but definite reproducible results for values in this region have not always been obtainable.

If the $-\log _{10} T$ scale is used, large uncertainties may thus be present if values of the order of $-\log _{10} T=2.0$ are being measured. Where possi- 
ble it would be preferable to adjust solutions so that absorbancies greater than 1.0 or 1.5 need not be measured.

The slit widths normally used on the Beckman spectrophotometer are believed to be less than those on any other commercial spectrophotometer. For spectral-transmission measurements these widths normally are such as to transmit from 0.5 to $1.5 \mathrm{~m} \mu$ of spectrum within the range 220 to 950 $\mathrm{m} \mu$ when the appropriate source and phototube are used. This feature of the instrument serves three useful purposes. It enables the spectral transmission of samples having high selectivity to be determined with an accuracy unattainable with instruments having wider slits. It makes possible the precise and accurate comparison of nearly identical samples, even those having high absorption, without the use of slits unduly wide. It makes possible the reliable determination of very low transmittances by a step-down procedure with samples of intermediate transmittance.

As with any spectrophotometer the wavelength calibration should be carefully checked when the instrument is received and as often thereafter as may be found necessary. The mercury green line at $546.1 \mathrm{~m} \mu$ has proved to be a good reference line for adjustment purposes. When this wavelength is correctly adjusted, errors in the rest of the scale exceeding $0.5 \mathrm{~m} \mu$ were not found on either of the instruments considered, except above $600 \mathrm{~m} \mu$, where the dispersion is relatively small.

As with any single-dispersion spectrophotometer stray-energy errors will usually be present in the regions of low spectral response. The filter supplied by the makers of the Beckman instrument should always be used in the region from 320 to $400 \mathrm{~m} \mu$ with incandescent source. Other filters may be desirable for certain types of transmission or for other special reasons. Multiple reflection effects should be considered if errors less than 1 percent are considered important.
The instrument is subject to error if a fluorescent sample is measured. The magnitude of this error will depend on the ratio of fluorescent to incident energy and on the inter-relation of the photocell sensitivity and the wavelength regions of excitation and fluorescence. If the fluorescent energy is in the visible region, it can be detected by placing the sample in the beam and viewing it from above as the wavelength is varied through the ultraviolet and visible regions. One can thus be forewarned. If the fluorescence is in the ultraviolet, such error would be difficult to detect without special equipment for the purpose. The error can be much greater in reflection than in transmission measurements.

Care should be taken in the measurement of the transmittance of polarizing materials. Two effects may be noted. As with all prism instruments the energy transmitted by the dispersing prism becomes partially polarized. As a result, the value of transmittance obtained will depend on the orientation of the polarizing sample being measured. A second and more serious effect may arise if the quartz plate over the entrance slit (and to a much less extent the quartz plate or lens over the exit slit) is cut with the optic axis parallel to the surfaces of the plate. Serious distortions of the transmittance curve may result, as shown in figure 4 .

In conclusion, therefore, it may be stated that spectral-transmission measurements of high reliability are obtainable on the Beckman quartz spectrophotometer. To obtain such results, however, one must take various precautions. The instrument is convenient and rapid to use and covers a wavelength range not heretofore readily available in a single instrument.

Washivgton, March 7, 1947. 